\title{
Extending the Algebras of Design
}

\author{
Iestyn Jowers ${ }^{1} \cdot$ Christopher F. $\operatorname{Earl}^{1}$
}

Published online: 25 July 2015

(C) Kim Williams Books, Turin 2015

\begin{abstract}
Algebras of design have previously been investigated for shapes composed of rectilinear geometric elements, such as lines and planes, and the properties of these algebras have been found to be beneficial for formalising designs, as well as the visual processes used by designers as they manipulate shapes in their design explorations. In this paper, an overview is presented of the application of these algebras in formalising design processes, and this is followed by a discussion concerning issues that arise when the algebras are extended to accommodate nonrectilinear designs, represented by shapes composed of curves, surfaces and solids. Consideration of non-rectilinear shapes introduces new problems not previously identified in the established formalism, resulting from the geometries and topologies of the shapes. These give rise to significant questions about the relationships between shapes and the property of embedding, which is fundamental to the construction of algebras of design.
\end{abstract}

Keywords Shape algebras · Shape grammars - Design representation

\section{The Boolean Algebra of a Design}

Pictorial representations, such as sketches and digital models, play an important role in architectural design for supporting creative processes of ideation and form development, as well as analysis and communication of developing design concepts. As a result, aspects of architectural design can be modelled according to shape

Iestyn Jowers

i.r.jowers@open.ac.uk

Christopher F. Earl

c.f.earl@open.ac.uk

1 Design Group, The Open University, Milton Keynes, UK

Birkhäuser 
computations (Stiny 2006), where shapes are abstractions of the pictorial representations that architects use to support their design processes. Shapes are formally defined according to finite arrangements of the geometric elements used to construct representations, such as points, lines, planes and solids, and can include labels or weights to model non-spatial information attributed to a design. For example, (Koning and Eizenberg 1981) presents an analysis of Frank Lloyd Wright's prairie style, in which designs are represented as shapes decomposed into Froebelean-type building blocks. The analysis resulted in a shape rule schemata that formalises the style according to spatial relations between volumes differentiated as functional zones, as illustrated in Fig. 1 for the design of the Robie house.

Identification of the parts of a design imposes a structure on the representative shape. This structure, defined according to the identified set of parts, corresponds to a Boolean algebra for a finite set and its subsets, and is partially ordered according to a part relation, closed over operations of sum and product, with the complete shape as unit, the empty shape as zero, and complements defined accordingly. The algebra enumerates potential decompositions of the shape according to the identified parts, and is equivalent to a complemented distributive lattice, as illustrated in Fig. 2. Here the main floor level of the design of the Robie house is decomposed according to functional zones, with the union of parts given by the supremum (join) and the intersection by the infimum (meet), and empty parts of the shape, represented by their boundaries, are included for legibility. This lattice illustrates a particular decomposition of the shape, and is indicative of a fixed symbolic representation according to the identified set of (subsets of) parts. But shapes are visual constructs, and the visual richness that is apparent in even the simplest shape cannot be reduced to a set of symbolic elements (Stiny 2006). Accordingly, the lattice illustrated in Fig. 2 gives only an account of a specific decomposition of the main floor level of the design of the Robie house, one defined according to functional zones, and does not represent the complete Boolean algebra embodied by the shape.

Shapes have no inherent parts and alternative analyses of the design of the Robie house would result in alternative decompositions. For example, consideration of room layout, instead of functional zones, would identify a different decomposition of the design with parts representing the rooms of the house. Similarly, analyses of the design according to performance factors such as ventilation or lighting, would

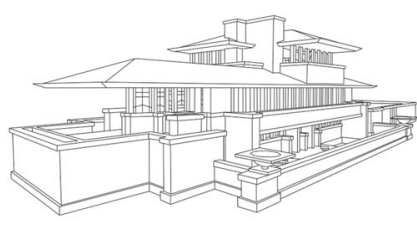

external view

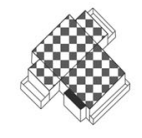

bedroom level

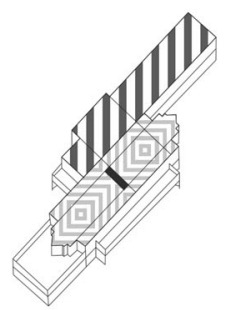

main floor level

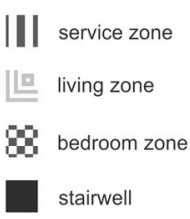

stairwell

Fig. 1 The Robie house, decomposed into functional zones, represented as Froebelean-type blocks. Image: authors, after (Koning and Eizenberg 1981) 


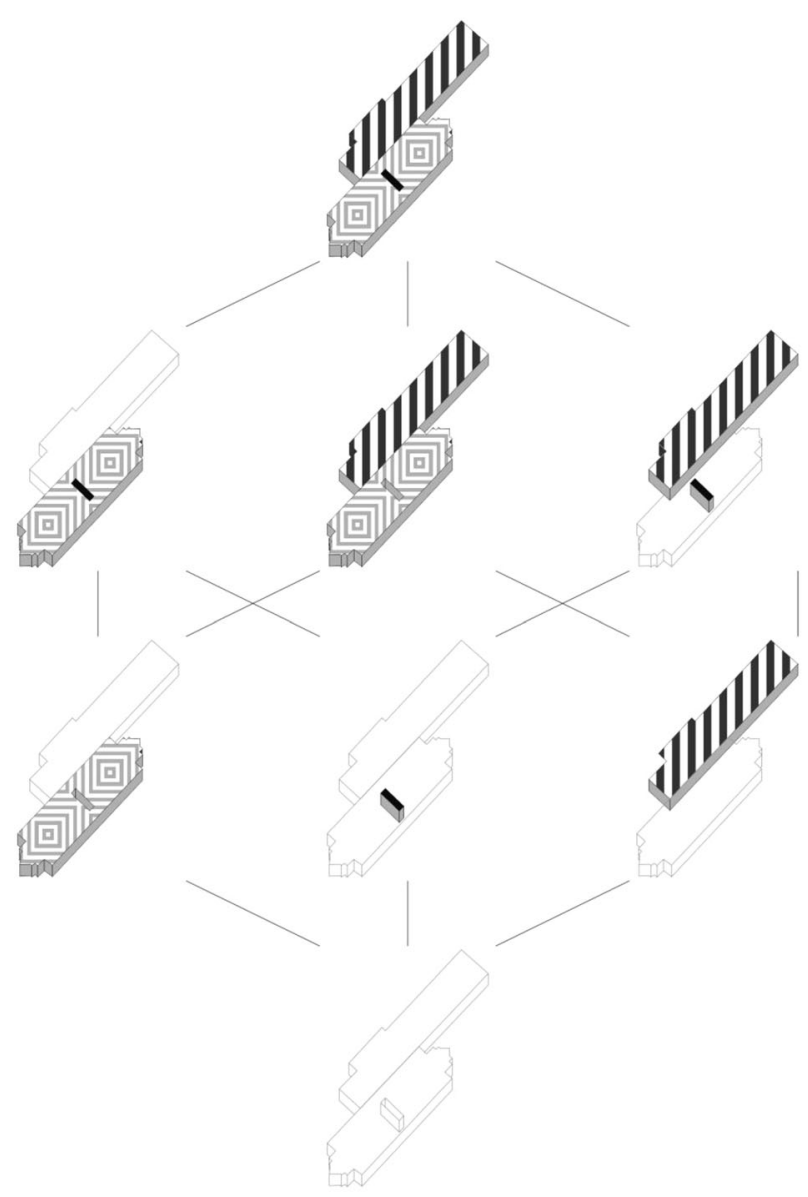

Fig. 2 A lattice of the main floor level of the design of the Robie house

suggest other decompositions of the shape, with parts identified according to corresponding metrics. Also, other decompositions can result from other processes that involve applying a description to the design, for example for the purposes of representation or communication (Krstic 2004). It is through these processes of enquiry and description that the structure of a shape is defined, and as a result shapes can be continuously reinterpreted to enable the different modes of interrogation and enquiry necessary to support a design process (Jowers and Earl 2012). With respect to the algebraic structure of a shape, this plethora of decompositions gives rise to a Boolean algebra which is partially ordered by the subshape relation, is closed over operations of sum and product, with the complete shape as unit and the empty shape as zero, but is infinite in breadth (Stiny 1990). Consequently design representations can be interpreted and structured according to any subshapes that are recognised as embedded parts, and the visual richness that is inherent in shapes can be utilised in computations within formal algebras. 
This account of shapes is analogous to mereological sums, which are defined according to part-whole relations, and do not decompose uniquely into parts (Casati and Varzi 1999). Indeed, there is a close connection between Boolean algebras and mereology, as explored in (Hovda 2009), although a direct isomorphism is not possible because mereological structures do not contain a zero element (Eberle 1970). In design, the zero is accounted for by the empty shape which is both philosophically and practically an essential element for realistically formalising design representations and the processes applied to them. Philosophically, the empty shape is the untouched medium: a blank sheet of paper, an empty CAD file, etc. Practically, it ensures closure of shape algebras under shape operations. As visual constructs, shapes also have other properties that differentiate them from mereological sums. In particular, the parts of a shape can be both timeless and temporary, and neither timeless nor temporary parts are adequately accounted for by standard mereological conceptions (Fine 1999). A shape's parts are timeless, because they are persistent components of the shape. The empty shape is the only shape that does not have any proper parts and removal of a part from any non-empty shape will result in that particular shape ceasing to exist. The parts can also be temporary, because at any particular moment a given part may not be recognised within the structure of the shape. For example, individual rooms are always a part of the design of the Robie house, but may not be recognised as such when the design is decomposed according to functional zones. The temporality of the parts of a shape plays an important role in creative design. It introduces a visual ambiguity that allows shapes to be more than externalisations of ideas; they are an active part of the design process. This is most obviously exemplified in sketching processes, where the sketching activity is not only directed by the architect, and her internal thought processes, but also by shapes recognised in the sketches (Schön and Wiggins 1992).

There is a reciprocal interaction between the architect and the representation, which results from seeing shapes in new ways and reinterpreting their structure according to alternative parts. This process involves not only decomposition of shapes, but also transformation via manipulation of recognised parts including components and shape elements, which are moved, added, deleted, stretched, reflected, etc., (Prats et al. 2009; Paterson and Earl 2010). These manipulations are formalised according to shape rules, which recognise parts of shapes under Euclidean transformations, and replace them under Boolean operations. Such rules implement computations in algebras which extend beyond those defined by particular shapes and their parts.

\section{The Algebras of Design}

In the previous section, a design representation was presented as a Boolean algebra defined over the representative shape and its embedded parts. This is a particular (static) view of design representation, which supports reinterpretation according to decompositions identified via enquiry and description. But, consideration of manipulations of representations in a design process forces this view to be expanded to include variations of the shape according to added and transformed 
parts. For example, representing the design of the Robie house according to Froebelean-type blocks gives rise to a Boolean algebra, which includes all possible decompositions of the shape into blocks, partially ordered by the subshape relation, with the complete shape as unit and the empty shape as zero. Manipulation of the design of the Robie house by adding more blocks or by transforming recognised parts of the design results in shapes which are not included in this algebra. Therefore, to ensure closure under such manipulations it is necessary to consider not only the shapes that are parts of the Robie house, but all shapes that are of potential interest, even shapes of which the Robie house is a proper part. This gives rise to an algebra of design, which includes all possible design representations composed of Froebelean-type blocks, partially ordered by the subshape relation (Stiny 2006). Other algebras of design can similarly be defined, and the simplest examples of these contain shapes composed of a single type of geometric element, (either points, lines, planes or solids) and are denoted by $U_{i j}(i \leq j)$, where $i$ indicates the dimension of the geometric element and $j$ represents the dimension of the Euclidean embedding space. For example, the Froebelean-type block representation of the design of the Robie house is in the algebra $U_{33}$, where volumes are arranged in 3D space. Also contained in this algebra are Froebelean-type block representations of all of Wright's Prairie house designs, as well as all other shapes defined by arrangements of volumes in 3D space. Similarly, designs represented in 2D sketches are in the algebra $U_{12}$, and 3D wire frame models are in $U_{13}$. More interesting design representations, composed of combinations of different types of shapes as well as other non-spatial information, are formalised in algebras which are defined by the Cartesian products of these simple algebras, in combination with algebras of labels, $V_{i j}$, and weights, $W_{i j}$ (Stiny 1991). For example, the representations of the design of the Robie house in Fig. 1 combine lines, planes, and weighted volumes in the algebra $U_{13} \times U_{23} \times \mathrm{W}_{33}$.

Unlike the algebra of a particular design representation, these more general algebras of design do not include a unit. The only exception is the algebra $U_{O O}$ which contains a shape composed of a single point, and is isomorphic to the algebra of Boolean logic, with the point acting as unit, and the empty shape as zero. In other algebras $U_{i j}(i, j \neq 0)$, the empty shape is zero, but the unit would be a universal shape, which would include all other shapes as parts, and would by definition violate the condition that shapes are of finite extent. Instead, the algebras of design $U_{i j}(i$, $j \neq 0$ ) form generalised Boolean algebras (Stiny 2006). These simple algebras, $U_{i j}$, have the property that all shapes within the algebras can be defined from a set of shapes, and the sum operation applied under transformation (Stiny 2006). For $U_{0 j}$, all shapes can be defined from a point, repeated under transformed sums; for $U_{1 j}$, all shapes can be defined from a line, repeated under transformed sums; for $U_{2 j}$, all shapes can be defined from a set of triangles, repeated under transformed sums; for $U_{3 j}$, all shapes can be defined from a set of tetrahedra, repeated under transformed sums.

As lattices the algebras are closed under union and intersection, but these are not complete because although every intersection is defined, infinite unions are not. Consequently they are relatively complemented distributive lattices, which are equivalent to Boolean rings, closed under sum and product (Birkhoff 1940). They 
are also closed under continuous spatial transformations, including solid-body and Euclidean transformations. As such the algebras formalise the shapes, shape operations and spatial transformations that architects utilise in manipulations of design representations. Aspects of design processes concerned with pictorial representations can therefore be formally defined as shape computations within these algebras (Prats et al. 2009; Paterson and Earl 2010).

In practice, a design process is unlikely to be formalised according to any single simple algebra, $U_{i j}$. It would, instead be formalised by a complicated Cartesian product incorporating many of the types of shape and non-spatial information that are used to inform and support the development of a design. Consideration of the representational enquiries and manipulations that take place throughout such a process gives rise to a subalgebra of the algebras of design. The extent of such a subalgebra cannot be defined prior to completion of a design process, without compromising the outcome. In some instances such a compromise is required, for example, as explored in (Dounas 2008) with respect to algebraic formalisations of building codes. However, in general, the constructivist nature of design processes mean that the subalgebra which formalises a particular process cannot be determined until after that process is complete (Stiny 1991). Stouffs and Krishnamurti (2007) illustrate the potential for this algebraic representation, when applied to data intended to support a design process and all the various actors and agents involved. The examples reported illustrate the advantages of algebras of design over the point-set formalisations of design representations which underlie computer-aided design (CAD). The most prominent of these is that the algebras support both a reductionist and a constructivist approach to design development. The reductionist approach is commonly seen in CAD and fixes the structure of a design representation. This is beneficial for later stages of a design process when decisions have been made and certain aspects of the design have been fixed (Stacey and Eckert 2003). However, it can be detrimental for creative design since it necessitates that architects anticipate all future ways in which the parts of a design representation will be viewed and manipulated as development continues. Conversely, a constructivist approach does not fix the structure, and instead the parts of a design can be freely interpreted throughout the design process (Jowers and Earl 2012). Under a constructivist approach a design representation can accommodate the needs of all actors and agents in a design process, with its structure continuously changing in accordance with processes of enquiry and description. Such reinterpretation is a vital element in the exploration of a design problem, and the development of a design solution, and is believed to be a decisive component of innovative design (Suwa 2003).

\section{Non-Rectilinear Embedding}

Despite the promise of algebras of design for supporting reinterpretation of design representations, there remain important technical issues which have not, to date, been investigated in sufficient detail. In particular, the (Stiny 2006) formulation of the algebras focuses on rectilinear shapes and takes advantages of the peculiarities 
of rectilinearity, with little consideration of general application to non-rectilinear geometric elements, such as the curves and surfaces used in the representation of the design of the David and Gladys Wright house in Fig. 3.

As discussed in (Jowers and Earl 2011), the most prominent of these peculiarities are concerned with the concept of embedding, which is the fundamental principle from which algebras of designs and shape computations are derived. The subshape relation, which applies a partial order over shape decompositions, is defined according to embedding:

A shape $A$ is defined to be a subshape of a second shape $B$, denoted $A \leq B$, if all the geometric elements of $A$ can be embedded in all the geometric elements of $B$.

Other shape operations build on this definition of subshape, including shape identity:

A shape $A$ is defined to be identical to shape to a shape $B$, denoted $A=B$, if both $A \leq B$ and $B \leq A$;

Shape union:

A shape $C$ is defined to be the union of two shapes $A$ and $B$, denoted $C=A+B$, if it is the least shape for which $A \leq C$ and $B \leq C$;

Shape intersection:

A shape $C$ is defined to be the intersection of two shapes $A$ and $B$, denoted $C=A \times B$, if it is the greatest shape for which $C \leq A$ and $C \leq B$;

And shape difference:

A shape $C$ is defined to be the difference of two shapes $A$ and $B$, denoted $C=A-B$, if it is the least shape for which $A=C+A \times B$.

The embedding properties of rectilinear shapes are peculiar because lines, planes, and rectilinear solids are self-similar to an infinite order. In contrast to this, the embedding properties of non-rectilinear spatial elements are more limited. For comparison, refer to Fig. 4, where the dominant design element of the David and Gladys Wright house, the circular arc, is compared to the straight line and the parabolic arc. A line can be embedded in any other line in an uncountable number of ways, so line $\mathrm{A}$ is part of line $\mathrm{B}$ under an infinite number of Euclidean transformations, and similarly line B is part of line A under an infinite number of
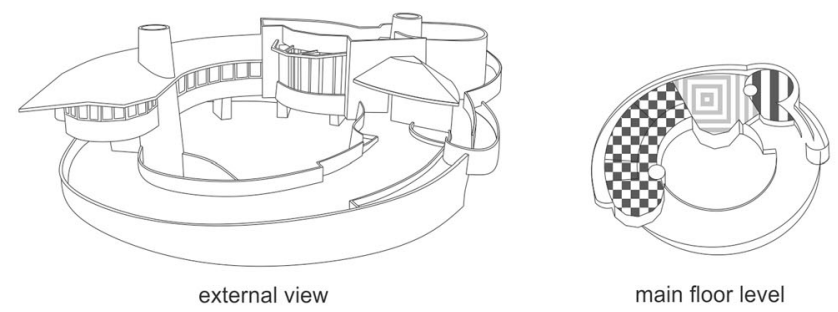

service zone

living zone

bedroom zone

Fig. 3 The David and Gladys Wright house, decomposed into functional zones 
transformations. In contrast, a circular arc can be embedded in a second arc in an uncountable numbers of ways, but only if the arc length of the second is greater than the arc length of the first. So, circular arc A is part of circular arc B under an infinite number of Euclidean transformations, but circular arc B is not part of circular arc A.

The embedding properties of parabolas are restricted further, and a parabolic arc can be embedded in a second arc in at most two ways, due to the reflective symmetry of parabolic arcs (Jowers and Earl 2011). So, parabolic arc A is part of parabolic arc B, under two Euclidean transformations, while parabolic arc B is not part of parabolic arc A. In essence, the curvature of the parabola serves to lock curve segments in place, thereby restricting its embedding properties. With respect to embedding, all lines are equal, but some curves are more equal than others.

Another peculiarity that arises when considering only rectilinear shapes is that there is little distinction between analytical and visual embedding. The properties of rectilinear geometric elements ensure that there is always a subshape relation between two elements of the same type, and that this relation is both visually and analytically correct. For non-rectilinear geometric elements, this is not always the case. For example, Fig. 5 illustrates how a curve segment can be visually embedded in many different curves. Analytically, this is not possible, since if two distinct curves are analytically described, (defined according to equations), then they can never have more than a finite number of intersection points. Analytical embedding will only map a curve segment onto other curve segments that have a common mathematical description. Under analytical embedding, the visually similar curve segments in Fig. 5 would all be distinct and could not be mapped on to each other despite their visual similarity. Krishnamurti and Stouffs (2004) advocate analytical embedding over visual embedding, because it results in unique shape descriptors which simplify computer implementation of shape computation. But, this is potentially at the expense of visual intuition, and may not accurately reflect the visual interaction between architects and pictorial representations of their designs. With respect to implementation, it is possible to utilise either analytical or visual

\begin{tabular}{|c|c|c|}
\hline Lines & Circular Arcs & Parabolic Arcs \\
\hline A
\end{tabular}

Fig. 4 Comparing embedding properties of lines, circular arcs and parabolic arcs

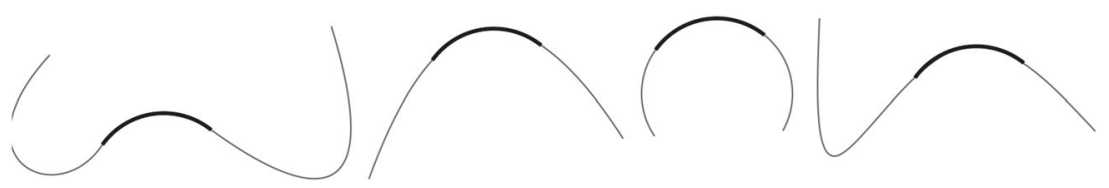

Fig. 5 Visual extensions of a curve segment 
embedding. For example, in (Jowers and Earl 2010), a method of implementing analytical embedding is presented, which builds on the intrinsic properties of parametric curves. Conversely, in (Jowers et al. 2010), a method of implementing visual embedding is presented, which builds on techniques of computer vision for object recognition in bitmap images. Both of these methods enable computation with non-rectilinear shapes, but the structures of the resulting algebras of design have not, to date, been investigated.

Given a specific design representation, for example the David and Gladys Wright house illustrated in Fig. 3, the algebra defined by decomposing the resulting shape into parts is the same as for rectilinear designs, as illustrated by the lattice in Fig. 6. The representative shape and its parts form a Boolean algebra, partially ordered by the subshape relation, and closed over operations of sum and product. The unit is defined by the complete shape, the zero by the empty shape, and the range of possible decompositions that arise in response to enquiry and/or description of the design representation gives rise to an algebra of infinite breadth. The fact that the parts include non-rectilinear geometric elements is of no consequence; regardless of whether embedding is analytical or visual, the parts are specified in relation to the

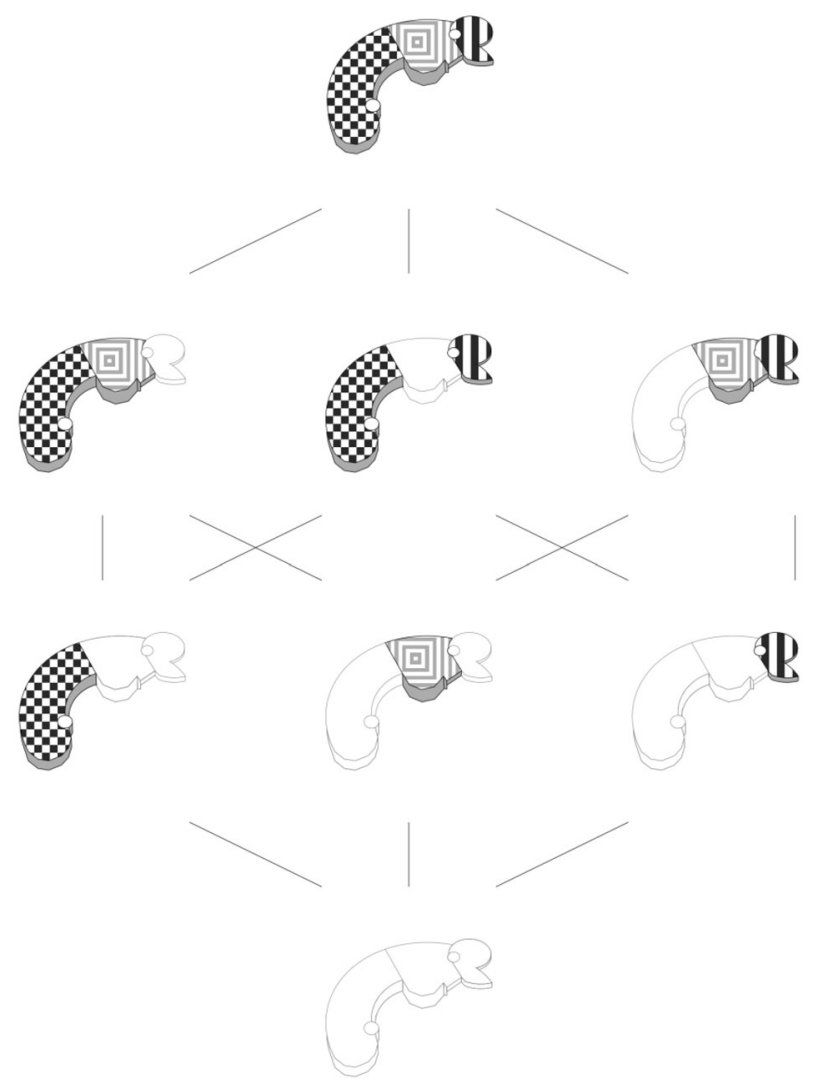

Fig. 6 A lattice of the main floor level of the design of the Robie house 
Fig. 7 A one-dimensional design composed of circular arcs

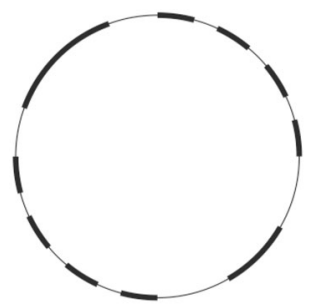

unit shape, and all joins and meets of the resulting complemented distributive lattice are uniquely defined. However, when considering the more general algebras of design, the fact that a shape includes non-rectilinear geometric elements is of significant consequence.

Consideration of non-rectilinear geometric elements introduces properties of shapes that need not be considered when geometric elements are rectilinear. For example, non-rectilinear geometric elements can exist without a boundary. For onedimensional elements these include circles, ellipses, and other closed curves, and analogous closed shapes exist for higher-dimensional non-rectilinear geometric elements, such as spheres. Other topologies also come into play, allowing holes in torii and other geometrical elements, according to their genus (Kelley 1991). As a result, the boundary of a shape can be the empty shape, a possibility not accounted for in formal treatments of shape boundaries, such as (Earl 1997), where it is argued that the only element with an empty boundary is the empty shape.

These different topologies have consequences beyond the geometric elements used to construct shapes; they also apply to the embedding spaces in which shapes are arranged. For example, consider a shape composed of circular arcs arranged in onedimensional space. The embedding properties of circular arcs mean that this shape cannot be embedded in a Euclidean one-dimensional space, such as a linear space; instead, the embedding space must be of the same type as the geometric elements; it must be a circle, as illustrated in Fig. 7. Because of the topology of this space, the properties of the resulting algebra of design are not the same as for shapes arranged in a Euclidean space. Specifically, the space is closed, which means the algebra is analogous to the algebra of a specific design representation: it corresponds to a Boolean algebra, partially ordered according by the subshape relation, closed over operations of sum and product, with the space (which is finite in extent) as unit, the empty shape as zero, and complements defined accordingly. A one-dimensional circular design might seem superficial in nature, but is indicative of the issues that arise when architects explore designs in different embedding spaces, for example when applying ornamental patterns to curved surfaces rather than flat surfaces.

\section{Surveying the Algebras of Curved Shapes}

The differences in embedding properties, between rectilinear and non-rectilinear geometric elements, mean that the properties of the resulting algebras of design are not well understood. If embedding is defined visually, then it is not clear what the resulting mathematical structures are. The concept of visual embedding is currently 
ill-defined, but is likely to involve some degree of approximation and visual tolerance, as explored in (Jowers et al. 2010) and (Keles et al. 2012). The mathematical precision that underlies the treatment of shapes outlined in (Stiny 2006), is lost, but with the benefit of algebras of design that better reflect architects' visual interactions with pictorial representations. One consequence of this visual 'fuzziness' is that under visual embedding non-rectilinear geometric elements cannot be uniquely identified, and a given curve can be embedded in many different types of curve, as illustrated in Fig. 5; parts cannot be uniquely identified, and the subshape relation does not give rise to a relatively complemented distributive lattice. The structure that does arise from considering visual embedding warrants further research, but the remainder of this discussion will focus on issues that arise as a result of analytical embedding.

If embedding is defined analytically, then non-rectilinear geometric elements can be uniquely identified according to mathematical descriptions; for example, a line can be uniquely identified according to a linear equation, a circular arc according to the equation of a circle, and a parabolic curve according to a parabolic equation. Parts can be uniquely specified, and the subshape relation establishes a relatively complemented distributive lattice with all finite joins and meets uniquely defined. The resulting algebras of design are therefore relatively complemented distributive lattices, which are equivalent to Boolean rings, closed under sum and product. However, unlike algebras with shapes composed of rectilinear geometric elements, the resulting structure does not correspond with visual intuition; for example the visually identical curve segments illustrated in Fig. 5 are distinct, and each is included in a different simple algebra.

The algebraic framework proposed in (Stiny 2006) is summarised in Fig. 7, where the simple algebras $U_{i j}$ are enumerated for $0 \leq i \leq 3$ and $i \leq j$. It is typically assumed, for example in (Stiny 2006) and in (Krishnamurti and Stouffs 2004), that shapes composed of non-rectilinear geometric elements, such as the curves and surfaces used in the representation of the design of the David and Gladys Wright house in Fig. 3, are included within this framework. But consideration of the embedding properties of non-rectilinear geometric elements suggests that this cannot be so. For example, an algebra of design that includes shapes composed of both rectilinear and non-rectilinear geometric elements cannot be one of the simple algebras included in Fig. 8. This is because, under analytical embedding, rectilinear and non-rectilinear geometric elements are always distinct, under Euclidean transformations. For example, a straight line cannot be embedded in a circular arc, nor can a circular arc be embedded in a straight line. Algebras that include shapes

Fig. 8 Algebras of design, from (Stiny 2006)

$$
\begin{aligned}
& U_{00} U_{01} U_{02} U_{03} \\
& U_{11} U_{12} U_{13} \\
& U_{22} U_{23} \\
& U_{33}
\end{aligned}
$$


composed of both rectilinear and non-rectilinear geometric elements must instead be composite algebras, with different types of geometric element combined under Cartesian products. For example, an algebra of design that includes shapes composed of lines and arcs is a Cartesian product of an algebra of design in which shapes are composed of lines, and of an algebra of design in which shapes are composed of circular arcs.

Similarly, an algebra of design that includes shapes composed of different types of non-rectilinear geometric elements cannot be a simple algebra. This is because, under analytical embedding, different types of non-rectilinear geometric elements are distinct. For example, a circular arc cannot be embedded in a parabolic curve, nor can a parabolic curve be embedded in a circular arc. Algebras that include shapes composed of different types of non-rectilinear geometric elements must also be composite algebras, with different types of geometric element combined under Cartesian products. For example, an algebra of design that includes shapes composed of parabolic curves and circular arcs is a Cartesian product of an algebra of design in which shapes are composed of parabolic curves, and of an algebra of design in which shapes are composed of circular arcs.

These examples indicate that the framework summarised in Fig. 8 is incomplete. Non-rectilinear geometric elements are not included in the algebras $U_{i j}(i \leq j)$, and neither are they included in some, as yet undefined simple algebra of non-rectilinear shapes. Instead, when embedding is defined analytically, there must be an infinite number of algebras. The simple algebras enumerated in Fig. 8 include rectilinear shapes embedded in Euclidean spaces of different dimensions, but in addition to these a complete framework should also include simple algebras for each different type of non-rectilinear geometric element. To achieve this, it is necessary to clarify formally what the type of a geometric element is. In (Krishnamurti and Stouffs 2004), type acts as a filter for distinguishing categories of shapes, for example lines from curves, planes from surfaces, etc., and it is suggested that the equations of a geometric element can serve this purpose. With respect to algebras of design, type should reflect the embedding properties of geometric elements, so that geometric elements are distinguished according to embedding. This would ensure that elements with distinct embedding properties compose shapes defined within distinct algebras. But, under this criterion, the equation of a geometric element is insufficient a definition of type. For example, although circular arcs and parabolic curves are distinct in terms of embedding, they can both be described according to quadratic equations. Instead, other descriptors are necessary for distinguishing between types of geometric elements according to embedding properties. For example, in (Jowers and Earl 2010) intrinsic properties are used to distinguish between geometric elements of different types.

Type is a difficult concept to define because it depends not only on shape, but also on embedding space and the set of transformations used. Shape computations are typically defined so that shapes are embedded in an Euclidean space and manipulated and compared according to Euclidean transformations. But, designers often have to work in a range of embedding spaces, for example the curved walls of the David and Gladys Wright house give rise to a curved embedding space in which various architectural elements need to be arranged. Similarly, designers use a range 
of transformations; for example, affine transformations are often used to visually replicate the effects of perspective.

An example of a non-rectilinear embedding space is illustrated in Fig. 7, where circular arcs are embedded in a circular space. This example is analogous to the algebra $U_{11}$ where line segments are embedded in a one-dimensional Euclidean space; a linear space. The geometric elements are of the same dimension as the embedding space and consequently the geometric elements are the same type as the embedding space and no other type is permissible. In both examples, the set of transformations that can be used to transform and manipulate geometric elements are constrained to translations within the space. In $U_{11}$ these are linear translations along the line; in the embedding space illustrated in Fig. 7 these are circular translations along the circle. The type of the embedding space influences the type of geometric elements and the set of transformations.

Figure 9 illustrates an example where the dimension of the embedding space is greater than the dimension of the geometric elements. Here, one-dimensional geometric elements, including a line, a parabolic arc, an elliptic arc and a circular arc, are embedded in a two-dimensional conic space. In a Euclidean embedding space, such as a plane, these geometric elements may be considered to be of different types, but in this conic embedding space applying a rotation allows them to transform into each other. In the conic embedding space, under a rotational transformation, the line, the parabolic arc, the elliptic arc and the circular arc are all the same type.

This example is indicative of the importance of transformation in identifying the type of geometric elements. The set of transformations allowed in a computation specifies the spatial relationships that are included within the definition of embedding. For example, for computations in a conic embedding space, rotations must be allowed in order for the line, the parabolic arc, the elliptic arc and the circular arc to be considered the same type. Similarly, in Euclidean two-dimensional space, if the set of transformations allowed in a computation includes the affine transformations then the line, the parabolic arc, the elliptic arc and the circular arc

Fig. 9 Rotation of a line in a conic embedding space

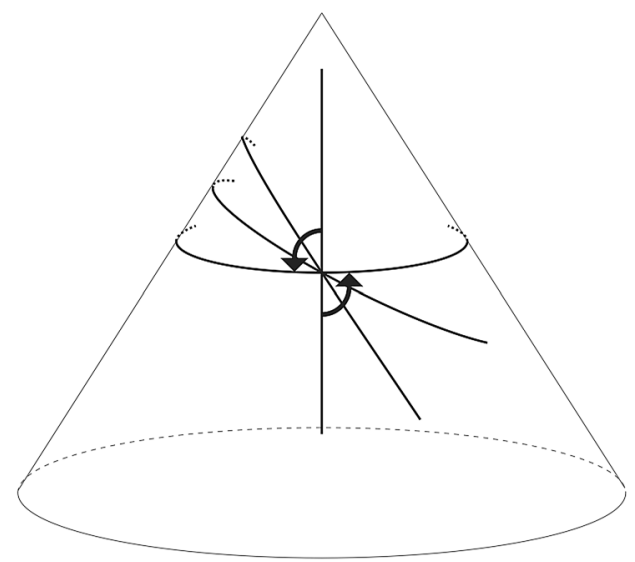


are all the same type, because they can all be mapped onto each other under affine transformations.

This brief discussion has highlighted some of the interesting properties that result when considering how the embedding relation applies to non rectilinear shapes, and the algebras of design that result from this. The examples explored suggest that the framework illustrated in Fig. 8 needs to be extended further to include different types of geometric elements as well as different types of embedding spaces. A complete framework would include all simple algebras differentiated according to the embedding properties of shapes and the spaces that they are arranged in. Computations within these new algebras aren't well understood, and further research is needed to explore the resulting structures.

\section{Concluding Remarks}

Modern CAD builds on a point-set representation of shapes which has proven effective for building design models of ever greater complexity, but has been found to be inadequate for supporting the visual shape manipulations that typify creative design (Stones and Cassidy 2010). One reason for this is because point-set representations are, by definition, represented symbolically in algebras $U_{0 j}$, and the visual properties of shapes in other algebras, $U_{i j},(i \neq 0)$, cannot be accommodated. The discussion presented in this paper has explored design representations which give rise to algebras of design, which are an elegant alternative to the point-set formalisations of design representations. These algebras build on a concept of embedding, which structures shapes according to identified parts that are appropriate for the task at hand, but do not fix this structure against future reinterpretation. Accordingly, they present a credible computational model for visual shape manipulations, and can be applied to formalise the shapes, shape operations and spatial transformations that architects utilise in manipulations of design representations. Theoretical development of the algebras of design have, to date, focused on points, lines, planes and solids, arranged in Euclidean space, and have paid little attention to the non-rectilinear forms and spaces that typify modern architecture. This discussion has explored how the current framework is insufficient to formalise these forms and spaces, and identified key areas for further investigation. Central to extending the algebras of design to accommodate nonrectilinear shape is formalising the concept of embedding. In the current framework it is unclear if embedding is an analytical relation or a visual relation, since for rectilinear shapes there is little difference between these. The discussion has highlighted that either choice results in complications which need to be resolved, either with the topological properties of geometric elements and the spaces in which they are embedded, or with more fundamental concepts of visual similarity, and how this is used by architects as they read, create, manipulate and describe shapes in their design processes. 


\section{References}

Birkhoff, G. 1940. Lattice Theory (3rd Edition). Rhode Island: American Mathematical Society.

Casati, R. and A. Varzi. 1999. Parts and Places: the structures of spatial representation. Cambridge: MIT Press.

Dounas, T. 2008. Algebras, Geometries and Algorithms, Or How Architecture fought the Law and the Law Won. In: First International Conference on Critical Digital: What Matters(s)? 111-114. http:// cumincad.scix.net/cgi-bin/works/Show?cdc2008_111. Accessed 7 Jul. 2015.

Earl, C. F. 1997. Shape boundaries. Environment and Planning B: Planning and Design 24(5): 669-687.

Eberle, R. 1970. Nominalistic Systems. Dordrecht: D. Reidel Publishing Company.

Fine, K. 1999. Things and their parts. Midwest Studies in Philosophy 23: 61-74.

Hovda, P. 2009 What is classical mereology? Journal of Philosophical Logic 38(1): 55-82.

Jowers, I. and C. Earl. 2010. The construction of curved shapes. Environment and Planning B: Planning and Design 37(1): 42-58.

Jowers, I. and C. Earl. 2011. Implementation of curved shape grammars. Environment and Planning B: Planning and Design 38(4): 616-635.

Jowers, I. and C. Earl. 2012. Shape Interpretation with Design Computing. In: Design Computing and Cognition'12, ed. J. S. Gero, 343-360. Dordrecht: Springer.

Jowers, I., D. C. Hogg, A. McKay, H. H. Chau and A. de Pennington. 2010. Shape Detection with Vision: Implementing Shape Grammars in Conceptual Design. Research in Engineering Design 21(4): 235-247.

Keles, H. Y., M. Özkar and S. Tari. 2012. Weighted shapes for embedding perceived wholes. Environment and Planning B: Planning and Design 39(2): 360-375.

Kelley, J. L. 1991. General Topology. New York: Springer.

Koning H. and J. Eizenberg. 1981. The language of the prairie-Frank Lloyd Wright's Prairie houses. Environment and Planning B: Planning and Design 8(3): 295-323.

Krishnamurti, R. and R. Stouffs. 2004. The boundary of a shape and its classification. Journal of Design Research 4(1).

Krstic, D. 2004. Computing with analyzed shapes. In: Design Computing and Cognition'04, ed. J. S. Gero, 397-416. Dordrecht: Kluwer Academic Publishers.

Paterson, G. and C. Earl. 2010. Line and Plane to Solid: Analyzing their use in design practice through shape rules. In: Design Computing and Cognition'10, ed. J. S. Gero (ed), 251-267. Dordrecht: Springer.

Prats, M., S. Lim, I. Jowers, S. Garner, S. Chase. 2009. Transforming shape in design: Observations from studies of sketching. Design Studies 30(5): 503-520.

Schön, D. A. and G. Wiggins. 1992. Kinds of seeing and their functions in designing. Design Studies 13: 135-56.

Stacey, M. and C. Eckert. 2003. Against Ambiguity. Computer Supported Cooperative Work 12: $153-183$.

Stiny, G. 1990. What is a design? Environment and Planning B: Planning and Design 17(1): 97-103.

Stiny, G. 1991. The algebras of design. Research in Engineering Design 2:171-181.

Stiny, G. 1994. Shape rules: closure, continuity, and emergence. Environment and Planning B: Planning and Design 21(7): 49-78.

Stiny, G. 2006. Shape: Talking about seeing and doing. Cambridge: MIT Press.

Stones, C. and T. Cassidy. 2010. Seeing and discovering: how do student designers reinterpret sketches and digital marks during graphical design ideation? Design Studies 31(5): 439-460.

Stouffs, R. and Krishnamurti, R. 2007. Sortal Structures: Supporting Representational Flexibility for Building Domain Processes. Computer-Aided Civil and Infrastructure Engineering 22: 98-116.

Suwa, M. 2003. Constructive perception: coordinating perception and conception toward acts of problem finding in a creative experience. Japanese Psychological Research 45: 221-234.

Iestyn Jowers (BSc, MSc Mathematics, PhD Design) is a Research Fellow in the Design Group at the Open University, UK. His primary area of research is in the role of shapes in design processes, including investigations into shape computations and shape grammars, generative design, additive manufacturing 
and computational making. Prior to joining the Open University in 2013 he worked as a post-doctoral research fellow at TU Munich, University of Leeds, and University of Cambridge.

Christopher F. Earl (BA, MSc Mathematics, PhD Design) is Professor of Engineering Design at the Open University, UK, since 2000. His primary area of research is in generative synthesis in Design, particularly relating to shape and function. His other research includes modelling and planning design processes. From 1991 to 2000 he was in the Engineering Design Centre, at Newcastle University, where his research concentrated on integration of design and manufacture, particularly in planning complex, engineer-to-order products. 